Ferrata Storti Foundation

\title{
ATR addiction in multiple myeloma: synthetic lethal approaches exploiting established therapies
}

\section{Oronza A. Botrugno, ${ }^{1}$ Silvia Bianchessi, ${ }^{2}$ Desirée Zambroni, ${ }^{3}$ Michela Frenquelli, ${ }^{1}$ Daniela Belloni, ${ }^{4}$ Lucia Bongiovanni, ${ }^{5}$ Stefania Girlanda, ${ }^{6}$ Simona Di Terlizzi, ${ }^{7}$ Marina Ferrarini, ${ }^{4}$ Elisabetta Ferrero, ${ }^{4}$ Maurilio Ponzoni, ${ }^{5}$ Magda Marcatti ${ }^{6}$ and Giovanni Tonon ${ }^{1,8}$}

Haematologica 2020

Volume 105(10):2448-2456

\section{Correspondence:}

GIOVANNI TONON

tonon.giovanni@hsr.it

Received: January 7, 2019.

Accepted: November 13, 2019.

Pre-published: November 14, 2019.

doi:10.3324/haematol.2018.215210

(C)2020 Ferrata Storti Foundation

Material published in Haematologica is covered by copyright. All rights are reserved to the Ferrata Storti Foundation. Use of published material is allowed under the following terms and conditions:

https://creativecommons.org/licenses/by-nc/4.0/legalcode. Copies of published material are allowed for personal or internal use. Sharing published material for non-commercial purposes is subject to the following conditions:

https://creativecommons. org//icenses/by-nc/4.0/legalcode, sect. 3. Reproducing and sharing published material for commercial purposes is not allowed without permission in writing from the publisher.
${ }^{1}$ Functional Genomics of Cancer Unit, Experimental Oncology Division, IRCCS San Raffaele Scientific Institute; ' ${ }^{2}$ aboratory of Lymphoid Organ Development, Division of Experimental Oncology, IRCCS San Raffaele Scientific Institute; ${ }^{3}$ ALEMBIC, Advanced Light and Electron Microscopy Bio-Imaging Center, IRCCS San Raffaele Scientific Institute; ${ }^{4}$ B-Cell Neoplasia Unit, Division of Experimental Oncology, IRCCS San Raffaele Scientific Institute; ${ }^{5}$ Pathology Unit, IRCCS San Raffaele Scientific Institute; ${ }^{6} \mathrm{Hematology}$ and Bone Marrow Transplantation Unit, IRCCS San Raffaele Hospital; ${ }^{7}$ FRACTAL, Flow Cytometry Resource Advanced Cytometry Technical Applications Laboratory, IRCCS San Raffaele Scientific Institute and ${ }^{8}$ Center for Omics Sciences, IRCCS San Raffaele Scientific Institute, Milan, Italy

\section{ABSTRACT}

Therapeutic strategies designed to interfere with cancer cell DNA damage response have led to the widespread use of PARP inhibitors for BRCA1/2-mutated cancers. In the haematological cancer multiple myeloma, we sought to identify analogous synthetic lethality mechanisms that could be exploited in established cancer treatments. The combination of ATR inhibition using the compound VX-970 with a drug eliciting interstrand cross-links, melphalan, was tested in in vitro, ex vivo, and most notably in in vivo models. Cell proliferation, induction of apoptosis, tumor growth and animal survival were assessed. The combination of ATM inhibition with a drug triggering double strand breaks, doxorucibin, was also analyzed. We found that ATR inhibition is strongly synergistic with melphalan, even in resistant cells. The combination was dramatically effective in targeting myeloma primary patient cells and cell lines by reducing cell proliferation and inducing apoptosis. The combination therapy significantly reduced tumor burden and prolonged survival in animal models. Conversely, ATM inhibition only marginally impacted on myeloma cell survival, even in combination with doxorucibin at high doses. These results indicate that myeloma cells extensively rely on ATR, but not on ATM, for DNA repair. Our findings postulate that adding an ATR inhibitor such as VX-970 to established therapeutic regimens may provide a remarkably broad benefit to myeloma patients.

\section{Introduction}

Inducing DNA damage in cancer cells for treatment purposes has been one of the mainstay in oncology for the past decades, and arguably remains one of the most effective strategies to induce cell death of epithelial and haematological cancers alike, to this day. ${ }^{1}$ Despite their effectiveness, one major limitation of the compounds eliciting DNA damage is represented by their poor specificity. ${ }^{1}$ Indeed, their administration quickly reaches dose-limiting side effects that are associated with unbearable toxicity. A very active research field is therefore aiming to identify synthetic lethal approaches, ${ }^{2,3}$ whereby genes and pathways within the DNA repair network are targeted to specifically increase the sensitivity of cancer cells endowed with specific genetic lesions, or towards DNA damaging agents. ${ }^{4}$ This quest has culminated in the identification of PARP inhibition as a means to trigger apoptosis in cancer cells presenting somatic or hereditary mutations in the BRCA1 and BRCA2 genes, ${ }^{5,6}$ which has profoundly modified the treatment of several tumor types, 
including breast and ovarian carcinomas. ${ }^{7}$ However, only a small subset of tumors, arising in specific tissues, present somatic mutations in BRCA1 or BRCA2 genes, where PARP inhibitors can be exploited. While cancers not bestowed with these mutations nevertheless may contain other genomic or molecular "BRCAness" signatures that make them sensitive to PARP inhibition, ${ }^{8}$ it is imperative to discover additional synthetic lethality strategies that can be deployed to improve the treatment and the outcome of cancer patients. Towards this goal, one of the most enticing paths calls upon the inhibition of specific genes implicated in DNA repair, to complement and synergize with established DNA damaging agents.

The vast majority of therapeutic regimens for the treatment of cancer patients include DNA damaging agents. The hematological cancer multiple myeloma (MM), is a particular case as it exhibits a still incurable clonal proliferation of malignant plasma cells. ${ }^{10}$ The alkylating agent melphalan was introduced in 1958 for the treatment of $\mathrm{MM}^{11}$ (later in association with prednisone), a landmark event in the history of the treatment of this disease, since there was no effective treatment for this cancer up to then. ${ }^{12}$ This treatment has remained the benchmark therapy for myeloma patients ever since. ${ }^{13}$ With regards to the mechanism of action of melphalan, it elicits cancer cell death by triggering interstrand DNA crosslinks (ICL), like other nitrogen mustards including chlorambucil and cyclophosphamide, still widely used for the treatment of various haematological cancers. ${ }^{4}$

The phosphoinositide 3-kinase (PI3K)-related kinases ATM and ATR control and coordinate the entire DNA damage response. ${ }^{14}$ ATM primarily orchestrates the global response to double-strand breaks (DSB). On the other hand, ATR is essential in relieving DNA replicative stress. ATR is endowed with an additional, less explored role, related to the repair of ICL, thus engaging the Fanconi anemia (FA) pathway. Therefore, ATM and ATR represent ideal candidates for targeted therapies aiming to unravel DNA repair in the presence of induced DNA damage. To this end, several ATM and ATR inhibitors have been recently developed. ${ }^{15,16}$

In this study, we comprehensively assessed the role of DNA damage response inhibition, namely of ATR and ATM, in MM, and analyzed if drugs, commonly used to treat $\mathrm{MM}$ patients, engage these pathways. We also assayed whether synthetic lethal approaches could be exploited, combining drugs used in the clinic, with ATM and ATR inhibition.

\section{Methods}

\section{MM cell lines and patient samples}

MM cell lines MM1.S, H929, KMS20, RPMI 8226, LP1, OPM2, U266, were kindly provided by fellow scientists or purchased from American Type Culture Collection (ATCC). Cell lines were authenticated by short tandem repeat (STR) analysis (Cell ID ${ }^{\mathrm{TM}}$ System, Promega, Madison, WI, USA) and routinely tested for the presence of mycoplasma contamination.

MM1.S-Luc and U266-Luc cells stably expressing luciferase were generated by transduction with a third generation lentiviral vector carrying the luciferase gene. pLenti PGK V5-LUC Neo (w623-2) was a gift from Eric Campeau (Addgene plasmid \# 21471).

Primary MM cells were collected from bone marrow (BM) aspi- rates through positive selection with anti-CD138 coated magnetic nanoparticles (Robosep, Stemcell Technologies, Vancouver, Canada). ${ }^{17}$ Samples from patients were obtained upon written informed consent. This study was carried out in accordance with protocols approved by the Institutional Review Board, and the procedures followed were in accordance with the Declaration of Helsinki of 1975, revised in 2000.

\section{Cell viability assays}

Cell viability was measured with CellTiter-Glo (Promega) according to the manufacturer's instructions. Luminescence reading was expressed as percentage relative to the DMSO or PBStreated control cells. The experimental data (percentage of viable cells compared to control) were analyzed independently using the Combenefit software. ${ }^{18}$

\section{Mice, bioluminescent imaging and pharmacological treatments}

All mice were housed and bred in the institutional pathogenfree animal facility, treated in accordance with the European Union guidelines and with the approval of the San Raffaele Scientific Institute Institutional Ethical Committee. Rag $2^{-/-} \gamma \mathrm{c}^{-1-}$ mice on a BALB/c background were kindly provided by CIEA (Central Institute for Experimental Animals, Kawasaki, Japan) and Taconic (Rensselaer, New York, NY, USA). Mice were injected intravenously with $5 \times 10^{6}$ luciferase expressing cells in $200 \mu \mathrm{L}$ of PBS and monitored for myeloma progression by bioluminescent imaging (BLI) using the IVIS SpectrumCT System (Perkin Elmer, USA).

Treatment commenced when the tumor burden became detectable. Mice were randomized into four treatment groups of five animals each. Treatment cycles consisted of 5 days of treatment followed by 2 days of rest. A total of three treatment cycles were given. VX-970, $60 \mathrm{mg} / \mathrm{kg}$ was administered by oral gavage once a day continuously for 5 days. Melphalan, $2 \mathrm{mg} / \mathrm{kg}$ was administered by intraperitoneal injection once a day on day 1,3 and 5 of each treatment cycle. Mice were euthanized by $\mathrm{CO}_{2}$ inhalation when they became detectably ill and developed hind limb paralysis.

\section{Immunohistochemistry on human BM biopsies and three-dimensional culture of primary cells}

Four $\mu \mathrm{m}$ thick sections were obtained from Bouin-fixed, paraffin-embedded tissue blocks of BM biopsies of untreated patients with a diagnosis of MM. Three-dymensional (3D) dynamic culture was performed using the RCCSTM bioreactor RCCS-1 (Synthecon Inc., Houston, TX, USA). ${ }^{17}$ Scaffold discs populated with MM cells were treated with either VX-970 (0.3 $\mu \mathrm{M})$ or melphalan $(1.2 \mu \mathrm{M})$ or both for 72 hours (h). At the end of the culture period, cells were recovered and stained with PC7-conjugated anti-CD38 (\#560677) and FITC-conjugated Annexin V (\#556547) both from BD Pharmingen (San Diego, CA, USA) before flow cytometric (FACS) analysis (FC500, Beckman Coulter, Brea, CA, USA). ${ }^{17}$

\section{Results}

\section{ICL-inducing melphalan activates the ATM and ATR pathways}

We first tested whether established DNA damaging agents used to treat MM patients activate the pathways commonly engaged in the DNA damage response. We first treated MM cells with melphalan. This alkylating agent triggers DNA ICL, which require ATR for their 


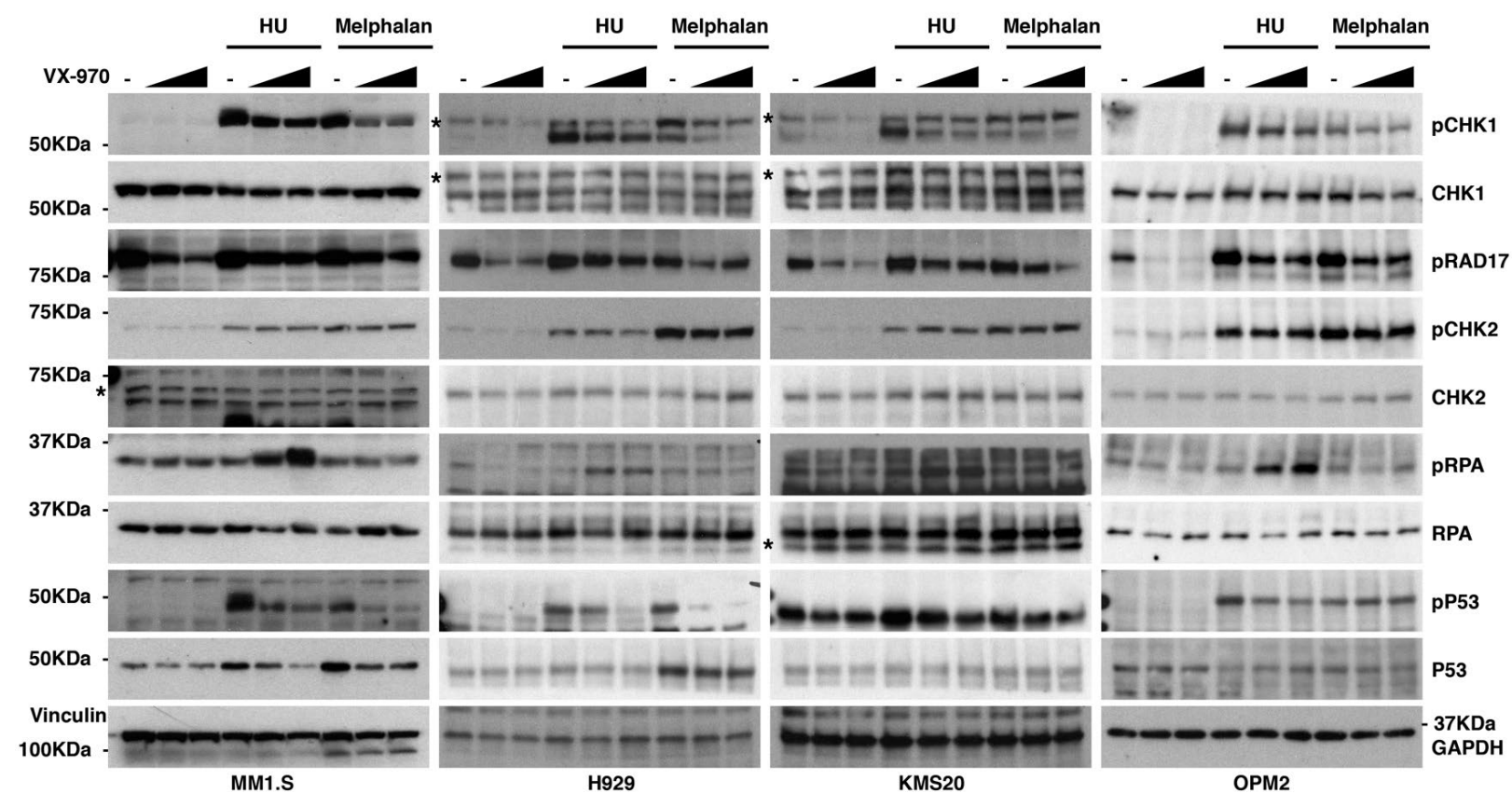

Figure 1. VX-970 selectively attenuates ATR-CHK1 signaling axis elicited by DNA damaging agents in multiple myeloma cells. Exponentially growing MM1.S, H929, KMS20 and OPM2 cells were either left untreated (NT) or treated with increasing concentrations of VX-970 (0.15 and $0.3 \mu \mathrm{M})$. Treatment with VX-970 was initiated 1 hour $(\mathrm{h})$ before the addition of hydroxyurea $(\mathrm{HU}, 2 \mathrm{mM})$ or melphalan $(50 \mu \mathrm{M})$. After $3 \mathrm{~h}$ cells were harvested and analyzed by immunoblotting for the expression of the indicated proteins. Vinculin for MM1.S and GAPDH for the other cell lines, were used as loading controls. The asterisks indicate unspecific bands.

resolution. ${ }^{4}$ As a control to assay the engagement of the ATR pathway, cells were also treated with hydroxyurea that elicits replicative stress, which also activates ATR (Figure 1). As cellular model systems, we tested several $\mathrm{MM}$ cell lines featuring ongoing DNA damage ${ }^{19}$ (Online Supplementary Figure $S 1 A-B)$. The treatment with melphalan triggered, as anticipated, DNA damage, assayed with $\gamma \mathrm{H} 2 \mathrm{AX}$, and elicited a DNA damage response, measured through the increase in total and phosphorylated P53 (Figure 1 and Online Supplementary Figure S2). Additionally, both the ATM and ATR pathways were engaged and activated, as demonstrated by the robust phosphorylation of their downstream main targets, pCHK2, and pCHK1 and pRAD17, respectively.

All together, these results suggest that ATR and ATM are actively engaged in the DNA damage response elicited by ICL in MM cells.

\section{ATR inhibition is strongly synergistic with melphalan}

We then assessed whether ATR inhibition impacted on the response of MM cells to melphalan. ATR inhibitors have been recently proposed as important cancer drug treatments. ${ }^{16}$ We used a novel derivative of the ATR inhibitor VE-821, VE-822 (also known as VX-970) ${ }^{20,21}$ in a panel of MM cell lines (Online Supplementary Figure S3 and Online Supplementary Table S1). We found that VX-970 was by far the most effective ATR inhibitor in reducing MM cells survival, when compared to VE-821, the compound that we previously tested ${ }^{22}$ and a recently reported structurally unrelated ATR inhibitor, AZD 6738. ${ }^{23} \mathrm{We}$ hence exposed several MM cell lines to increasing concentrations of VX-970, with or without melphalan. ATR inhibition reduced the overall levels of DNA damage and the DNA damage response triggered by melphalan. Along similar lines, CHK1 and RAD17 phosphorylation as well as $\gamma \mathrm{H} 2 \mathrm{AX}$ were markedly reduced when combining the ICL-inducing compounds with VX-970, while pCHK2 levels were unchanged (Figure 1 and Online Supplementary Figure S2).

We then explored whether ATR inhibition could synergize with the ICL-inducing compound melphalan. VX-970 alone consistently reduced cellular proliferation (Figure 2 A, dotted vertical line). Strikingly, this effect was profoundly enhanced when VX-970 was combined with melphalan (Figure $2 \mathrm{~A}-\mathrm{B}$ and Online Supplementary Table S2).

Specifically, some cell lines (MM1.S, NCI-H929) were sensitive to either melphalan or VX-970, and yet the combination of VX-970 with melphalan was highly synergistic, as revealed by the Bliss and Loewe synergy scores calculated using the Combenefit software ${ }^{18}$ (Figure 2A-B, Online Supplementary Figure S4 and Online Supplementary Table S2).

Other cell lines (KMS20 and RPMI 8226) were in general resistant to melphalan, as higher doses were required to obtain some reduction in proliferation (Figure 2A-B, middle panels, Online Supplementary Figure S4 and Online Supplementary Table S2). Notwithstanding, these cells responded well to VX-970. Furthermore, a strong synergy between melphalan at higher doses and VX-970 was also evident (Figure 2B and Online Supplementary Figure S4).

Finally, MM cell lines LP1 and OPM2 were resistant to melphalan, even at very high doses (Figure 2A-B, right panels, Online Supplementary Figure S4 and Online Supplementary Table S2), but remained sensitive to VX-970 alone, which was thus able to overcome melphalan resistance.

In a parallel set of experiments, we assessed whether this reduced proliferation was associated with increased 

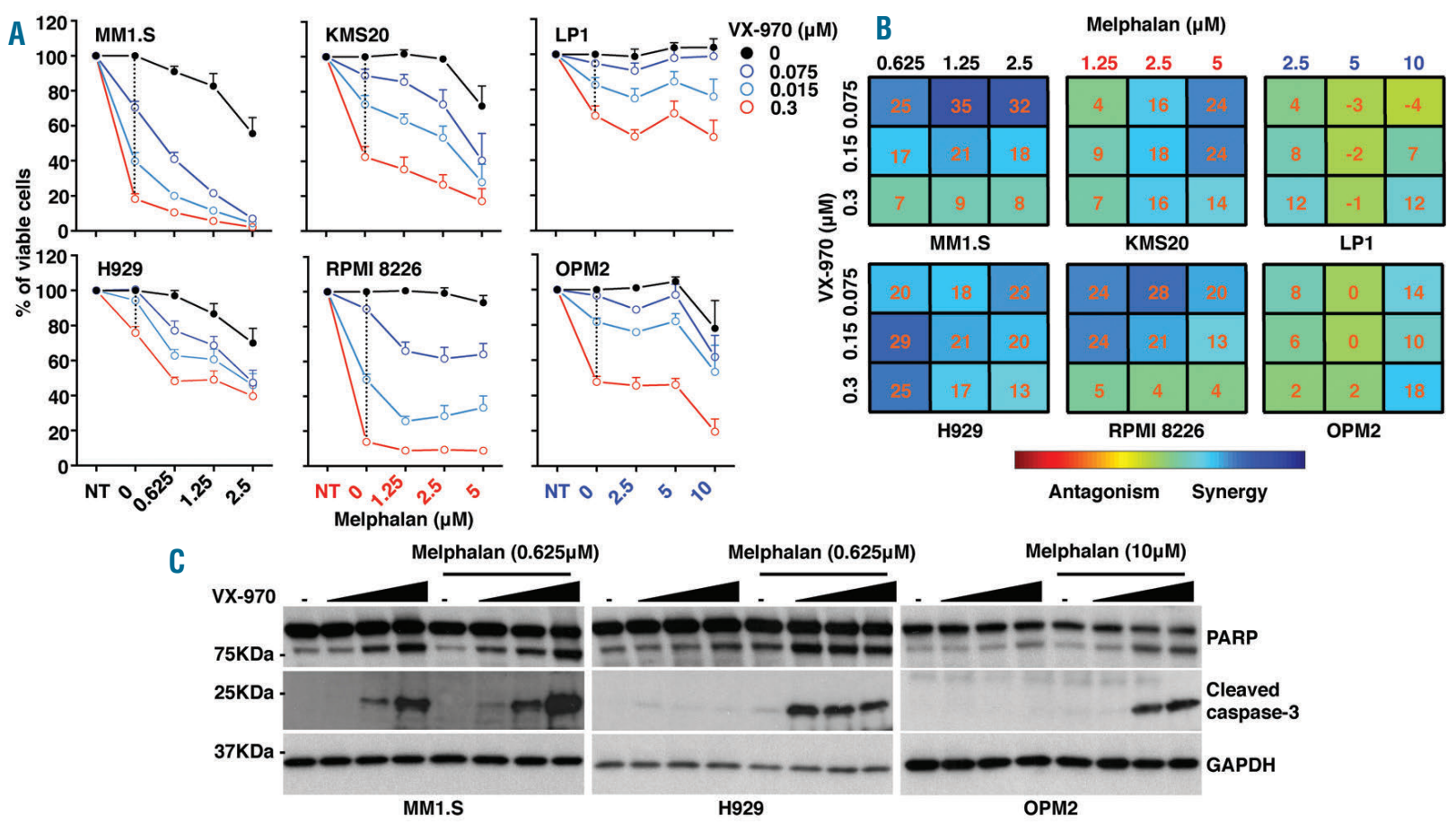

Figure 2. ATR inhibition by VX-970 sensitizes multiple myeloma cells to melphalan. (A) Multiple myeloma (MM) cells were seeded in 96-well plates and treated for 72 hours (h) with DMSO (as a control, untreated [NT]) or increasing concentrations of VX-970 either alone or in combination with the indicated doses of melphalan. Cell viability was assessed using CellTiter-Glo assay. Results are presented as the mean percentage of viable cells in treated samples, relative to DMSO control cells averaged from a minimum of three independent experiments (mean \pm standard error of the mean [SEM]), each with three repetitions per condition. Proliferation curves for each cell line were generated using GraphPad Prism. The dotted vertical line indicates the response of MM cells to increasing concentrations of VX-970 alone. Filled black dots indicate the cellular response to melphalan alone. Results of the statistical analysis are reported in the Online Supplementary Table S2. We could not rule out the potential appearance of general toxicity at the highest concentrations used. (B) For each cell line the Bliss synergy matrices and the relative drug synergy scores were calculated using the Combenefit software. The colored areas in the matrix are indicative of the degree of synergy between the drug combinations. (A-B) Different color codes (black, blue and red) are indicative of the concentrations of melphalan used to treat the cells depending on their sensitivity to the drug. (C) MM1.S and H929-melphalan sensitive and OPM2-melphalan resistant cell lines were treated with the indicated concentrations of melphalan either alone or in combination with increasing concentrations of VX-970 $(0.075,0.15$ and $0.3 \mu \mathrm{M})$. After $48 \mathrm{~h}$, cells were harvested, and immunoblotted for the indicated antibodies. The levels of cleaved PARP and caspase-3 served as indicators of apoptosis. GAPDH was used as loading control.

apoptosis, as assayed with PARP and caspase-3 cleavage (Figure 2C) and quantification by FACS analysis of Annexin V and PI positive cells (Online Supplementary Figure S5). The combination of VX-970 with melphalan triggered a robust apoptotic response (Figure 2C and Online Supplementary Figure S5).

Taken together, these results suggest that the combination of an ATR inhibitor alongside a DNA damaging agent eliciting ICL such as melphalan could represent a powerful, very effective drug combination to treat even resistant MM cells.

\section{ATM inhibition does not enhance apoptosis triggered by DSB-inducing compounds}

We then explored whether ATM inhibition could similarly impact on the survival and proliferation of MM cells. We first assessed whether a compound triggering DSB, such as doxorubicin, was able to activate the ATM pathway in MM cells. Indeed, the treatment with this compound elicited a strong activation of the ATM network in MM cells, as shown by the phosphorylation of its downstream targets CHK2 and $\gamma \mathrm{H} 2 \mathrm{AX}$ (Figure 3A and Online Supplementary Figure S6). These results suggest that, as expected, DSB call upon ATM for assisting on DNA repair. Treatment of $\mathrm{MM}$ cells with both doxorubicin and a broad ATM inhibitor, KU-55933, ${ }^{24}$ markedly reduced the activation of DNA damage response (as assessed with pCHK2 and $\gamma \mathrm{H} 2 \mathrm{AX}$ ) (Figure 3A and Online Supplementary Figure S6).

We then tested whether ATM inhibition, alone or in combination with doxorubicin, might restrain proliferation and elicit apoptosis. To our surprise, unlike VX-970, the treatment with KU-55933 was overall neither associated with a remarkable reduction in proliferation nor with apoptosis, even when used at very high concentrations (Figure 3B, Online Supplementary Table S3 and Online Supplementary Figure S7). Also, in all cell lines, the combination of doxorubicin and KU-55933 was not synergistic (Figure 3C and Online Supplementary Figure S8).

These results suggest that in MM cells the inhibition of ATM is not crucial for the survival after ongoing or induced DSB, unlike ATR inhibition after ICL-inducing treatments.

We also assayed MM cell lines for their sensitivity to both ATR and ATM inhibitors. In line with a recent report, ${ }^{25}$ we found that the combination of the ATR and ATM inhibition, that is, VX-970 and KU-55933, was synergistic, in some, but not all, MM tested cell lines (Online Supplementary Figure S9A-B and Online Supplementary Table S4). Specifically, ATM inhibition was able to further 


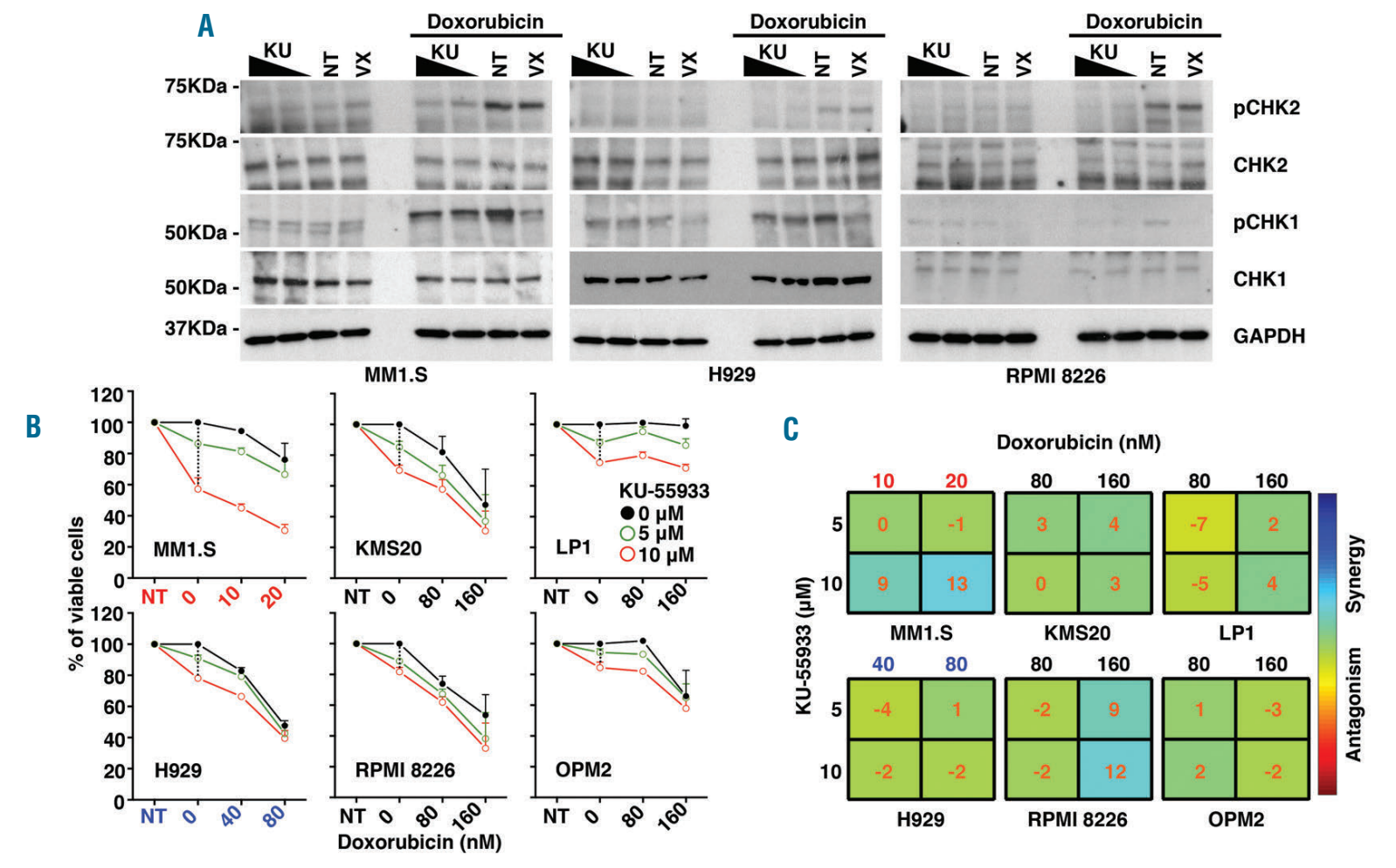

Figure 3. ATM inhibition by KU-55033 alone or in combination with doxorubicin does not restrain proliferation in multiple myeloma cells. (A) Exponentially growing MM1.S, H929 and RPMI 8226 cells were either left untreated (NT) or treated with increasing concentrations of KU-55933 (KU, 5 and 10 uM) and VX-970 (VX, 0.15 $\mu \mathrm{M})$. Treatment with both compounds initiated 1 hour $(\mathrm{h})$ before the addition of doxorubicin $(0.5 \mu \mathrm{M})$. After $3 \mathrm{~h}$, cells were harvested and and the expression of the indicated proteins was analysed by immunoblotting. GAPDH was used as loading control. (B) MM cells were seeded in 96-well plates and treated for $72 \mathrm{~h}$ with DMSO (as a control, NT) or increasing concentrations of KU-55933 either alone or in combination with the indicated doses of doxorubicin. Cell viability was assessed using CellTiter-Glo assay. Results are presented as the mean percentage of viable cells in treated samples, relative to DMSO control cells averaged from a minimum of three independent experiments (standard error of the mean (SEM), each with three repetitions per condition. Proliferation curves for each cell line were generated using GraphPad Prism. The dotted vertical line indicates the response of MM cells to increasing concentrations of KU-55933 alone. Filled black dots indicate the cellular response to doxorubicin alone. Results of statistical analysis are reported in the Online Supplementary Table S3. (C) For each cell line the Bliss synergy matrices and the relative drug synergy scores were calculated using the Combenefit software. The colored areas in the matrix are indicative of the degree of synergy between the drug combinations. (B-C) Different color codes (red, blue and black) are indicative of the concentrations of doxorubicin used to treat the cells depending on their sensitivity to the drug.

increase the already potent activity of VX-970, suggesting that ATR synergizes with ATM in repairing DSB in MM cells, despite having only a modest effect on the intensity and number of $\gamma \mathrm{H} 2 \mathrm{AX}$ foci per nucleus (Online Supplementary Figure S1OA-B).

\section{ATR inhibition enhances the therapeutic efficacy of melphalan in vivo}

To determine the effect of the combined treatment of VX-970 with melphalan in a more physiological setting, we assessed the activity of this combination in an in vivo

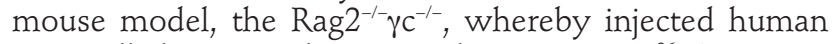
$\mathrm{MM}$ cells home to the mouse bone marrow. ${ }^{26}$ Treatment with melphalan, VX-970, or both drugs combined, was started at the fourth week after intravenous injection of the MM cells and continued for 3 weeks, using drug dosages reported in the literature ${ }^{21,27}$ (Figure 4A). No significant body weight loss was observed in the treatment groups, indicating that the treatment was well tolerated by the animals (data not shown). The tumor burden was assessed by in vivo imaging every week (Figure 4B). The combination of the two drugs was remarkably effective, profoundly restraining the rate of tumor development (Figure 4B).

We next assessed the impact of the treatments on survival (Figure 4 C). Both melphalan or VX-970 were able by themselves to delay the progression of the disease. Of note, VX-970 was more effective than melphalan, with a median survival of 67 days, instead of 57 days for the mice treated with melphalan, and 50 days for mice treated with vehicle alone. Strikingly, combining melphalan and VX-970 exerted a profound effect on the survival of the mice, with a median 107 day survival of the mice examined.

Taken together, these results suggest that the combination of an ATR inhibitor, VX-970, with a drug eliciting ICL, such as melphalan, is profoundly effective against MM cells presenting ongoing DNA damage.

The combination of ATR inhibitors and DNA damaging agents is effective also in MM cells with low levels of DNA damage

We have recently shown how the tumor cells of a subset of MM patients present with ongoing replicative stress, DNA damage and enhanced chromosomal instability. ${ }^{22}$ These patients have poor prognosis. Also, our previous data have shown how MM cell lines presenting with enhanced replicative stress are sensitive to the ATR inhibitor VE-821. ${ }^{21,22}$ Of note, one MM cell line, U266, displays lower levels of ongoing DNA damage, ${ }^{19,25,28}$ replicative stress, ${ }^{22}$ and responds less to these drugs. ${ }^{22}$

In line with the experiments performed in $\mathrm{MM}$ cells 
A

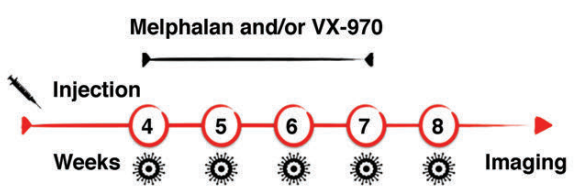

B

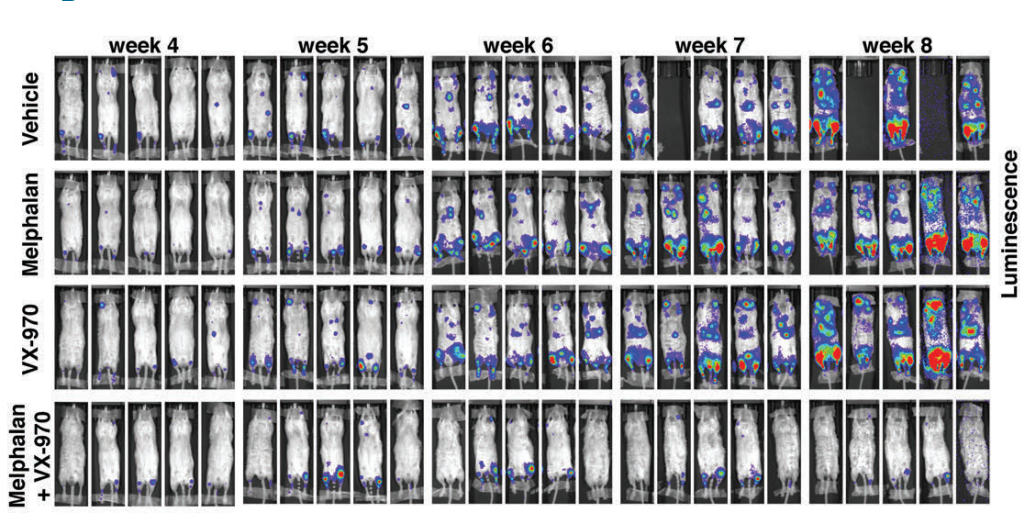

C

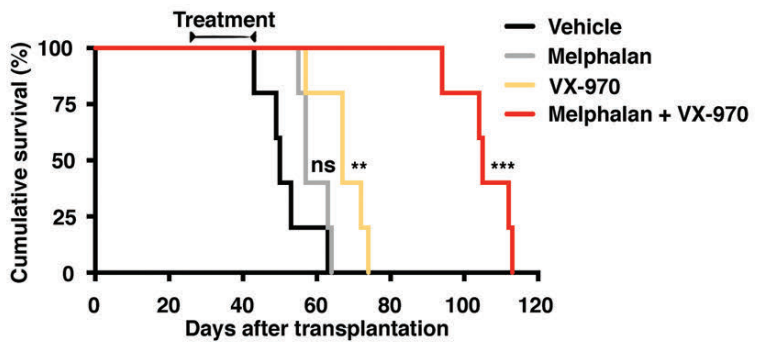

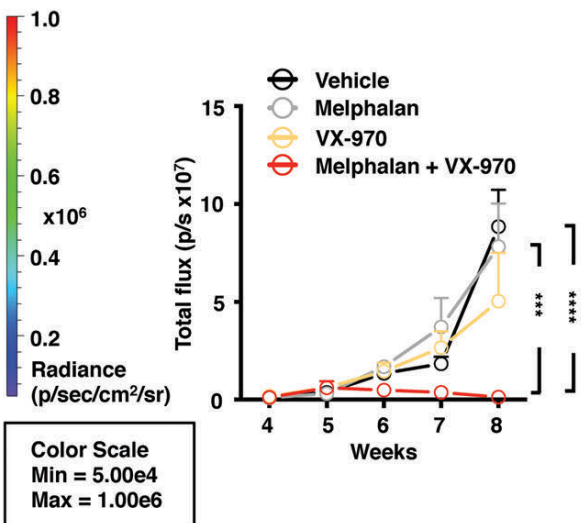

Figure 4. VX-970 is effective as monotherapy and enhances the therapeutic efficacy of melphalan in an orthothopic mouse model of multiple myeloma. (A) Rag $2^{-1-} \gamma \mathrm{C}^{-/-}$mice were injected intravenously with $5 \times 10^{6}$ MM1.S-Luc cells. The treatment started 4 weeks after injection, when the tumor burden became evident by bioluminescent imaging (BLI) (week 4). Mice were randomized into four treatment groups of five animals each: vehicle controls, melphalan 2 mg/kg, VX-970 60 $\mathrm{mg} / \mathrm{kg}$, or both. (B) BLI was performed every week during treatment (week 5 , week 6 and week 7 ) to monitor tumor burden and one week after the stop of the treatment (week 8). On the left, representative IVIS SpectrumCT System images of the luciferase signals observed in mice of each treatment group taken at the indicated interval are shown. On the right, the graph shows the tumor burden increase quantified as total flux measured from bioluminescent images during the treatment period. Data represent the mean \pm standard deviation (SD) of five mice in each treatment arm. Statistical analysis was performed by the two-way ANOVA and Tukey's multiple comparison test $(* * * P<0.0005, * * * * P<0.001)$. (C) Cumulative survival in each treatment arm was compared by Kaplan-Meier survival analysis. Statistical analysis was performed by log rank Mantel-Cox test $(* * P<0.01, * * * P<0.001)$.

with rampant extent DNA damage, we first assessed whether melphalan and hydroxyurea treatment elicited DNA damage and the engagement of the DNA repair pathway also in the U266 cell line. We found that indeed upon treatment with hydroxyurea and melphalan, several markers of the DNA repair pathway, including phosphorylation of $\mathrm{P} 53$ and of $\mathrm{CHK} 1$ were activated in this cell line as well (Figure 5A). In line with the previous experiments, the treatment of U266 cells with VX-970, when combined with hydroxyurea or melphalan, hampered the ATR response, as revealed by the reduction of pCHK1, pRAD17 and $\gamma \mathrm{H} 2 \mathrm{AX}$ protein levels (Figure 5A-B).

We then tested whether the combination of melphalan and VX-970 could be effective also in these cells. ATR inhibition by itself was moderately effective in reducing proliferation in U266 cells (Figure 5C and Online Supplementary Table S2), in line with our previous observations. ${ }^{22}$ The two drugs however were highly synergistic (Figure 5C and Online Supplementary Table S2). Along these lines, also in U266 cells the combination of VX-970 with melphalan triggered a robust apoptotic response as assayed through the analysis of PARP and caspase- 3 cleavage (Online Supplementary Figure S11A) and quantification by FACS analysis of Annexin V and PI positive cells (Online Supplementary Figure S11B).

In line with the experiments performed on $\mathrm{MM}$ cell lines presenting with intense ongoing DNA damage, also in U266 cells the combination of doxorubicin with the ATM inhibitor KU-55933 had neither a major effect on survival (Online Supplementary Figure S12A-D and Online Supplementary Table S3), nor was there any synergy between VX-970 and the ATM inhibitor KU-55933 (Online Supplementary Figure $S 9 A-B$ and Online Supplementary Figure S10A-B).

We also assessed the effect of VX-970 and melphalan, alone or in combination, in the Rag $2^{-1-} \gamma \mathrm{c}^{-1-}$ mouse model (Online Supplementary Figure S13A). These experiments confirmed that the combination was highly effective in hampering the growth of U266 MM cells in vivo (Figure 5D and Online Supplementary Figure S13B).

Taken together, these data suggest that even MM cells not presenting with DNA damage are sensitive to the combination of an ICL-inducing drug such as melphalan and ATR inhibition. ATM inhibition again had no major impact on cell survival upon induction of DSB.

\section{ATR inhibition synergizes with melphalan by inducing apoptosis in MM patient cells}

We then assessed the efficacy of this treatment strategy on MM patient cells, co-cultured with BMSC (BM stromal cells) in a 3D bioreactor system, which we recently reported as a sensitive and reliable method to assess drug response in $\mathrm{MM}$ (Figure 6A). ${ }^{17}$ While single treatments with either VX-970 or melphalan in primary MM cells was variably effective, the combination consistently elicited apoptosis, suggesting it may represent an promising, broadly active drug regimen in MM patients (Figure $6 \mathrm{~B}-\mathrm{C})$. Moreover, we analyzed by immunohistochemistry 

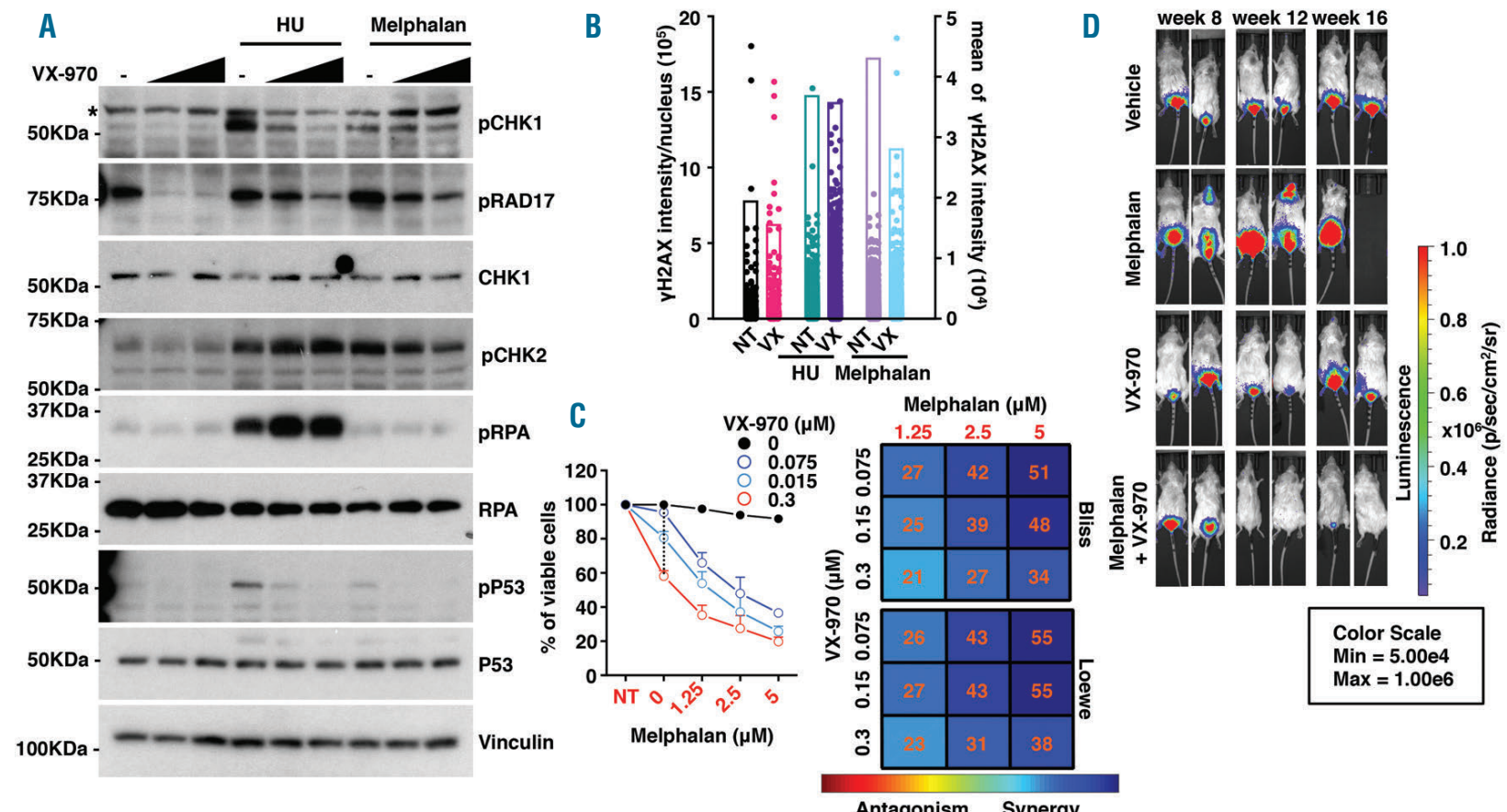

Figure 5. ATR inhibition by VX-970 sensitizes U266 cells to melphalan. (A) Exponentially growing U266 cells were either left untreated or treated with the indicated concentrations of VX-970. Treatment with VX-970 initiated 1 hour $(\mathrm{h})$ before the addition of hydroyurea $(\mathrm{HU}, 2 \mathrm{mM})$ or melphalan $(50 \mathrm{uM})$. After $3 \mathrm{~h}$ cells were harvested and analyzed by immunoblotting for the expression of the indicated proteins. Vinculin was used as a loading control. The asterisk indicates an unspecific band. (B) As in (A), cells were left untreated (NT) or treated with $0.15 \mu \mathrm{M}$ VX-970 (VX) before the addition of HU ( $2 \mathrm{mM})$ or melphalan (50 $\mu \mathrm{M})$ for $3 \mathrm{~h}$. At the end of the treatment, cells were fixed and processed for imaging flow cytometry to detect the $\mathrm{yH} 2 \mathrm{AX}$ signal for individual nucleus (left $\mathrm{Y}$ axis). The mean of $\gamma \mathrm{H} 2 \mathrm{AX}$ signal (right $Y$ axis) for each treatment is reported. Results are representative of two independent experiments. (C) Cells were seeded in 96-well plates and treated for 72 $\mathrm{h}$ with DMSO (as a control, NT) or increasing concentrations of VX-970 either alone or in combination with the indicated doses of melphalan. Cell viability was assessed using CellTiter-Glo assay. Results are presented as the mean percentage of viable cells in treated samples, relative to DMSO control cells averaged from a minimum of three independent experiments (mean \pm standard error of the mean [SEM]) each with three repetitions per condition. Proliferation curves on the left were generated using GraphPad Prism. The dotted vertical line indicates the response of the cells to increasing concentrations of VX-970 alone. Filled black dots indicate the cellular response to melphalan alone. The Bliss and the Loewe synergy matrices and the relative drug synergy scores calculated using the Combenefit software are reported on the right of the proliferation profile. The colored areas in the matrix are indicative of the degree of synergy between the drug combinations. Results of statistical analysis are reported in the Online Supplementary Table S2. (D) Rag $2^{-/-\gamma \mathrm{C}^{-/-}}$mice were injected intravenously with $5 \times 10^{6} \mathrm{U} 266-\mathrm{Luc}$ cells. The treatment started 8 weeks after injection, when the tumor burden became evident by BLI (week 8). Mice were randomized into four treatment groups of five animals each: vehicle controls, melphalan $2 \mathrm{mg} / \mathrm{kg}$, VX-970 $60 \mathrm{mg} / \mathrm{Kg}$, or both. Treatments were scheduled as reported in the Online Supplementary Figure S10A. BLI was performed every week during the treatment to monitor the tumor burden. Representative IVIS SpectrumCT System images of the luciferase signals observed in mice of the treatment groups taken before beginning of the treatment (week 8) and 1 and 5 weeks after the stop of the treatment (week 12 and week 16).

the BM biopsies of these MM patients for $\gamma \mathrm{H} 2 \mathrm{AX}$ and pCHK1 expression. Phosphorylated CHK1 was diffusely expressed in $80-90 \%$ of neoplastic cells, while $5-30 \%$ of tumor plasma cells expressed $\gamma \mathrm{H} 2 \mathrm{AX}$ ranged between $5-30 \%$ of tumor plasma cells, suggesting that the ATR pathway is predominantly activated in MM cells present pervasive activation of the ATR pathway and respond to the combination of VX-970 with melphalan independently from their basal levels of ongoing DNA damage.

\section{Discussion}

In this study, we have shown that drugs that elicit DNA damage and are commonly used to treat MM patients trigger a high activation of DNA repair response, engaging both the ATM and the ATR pathways. Surprisingly, however, ATM inhibition had little, if any, effect on MM cells, either used alone, or in combination with doxorubicin, a drug that elicits DSB. Conversely, inhibition of ATR was strongly synergistic with melphalan, a nitrogen mustard that has been used to treat MM patients for the past 60 years, and that induces ICL. Furthermore, ATR inhibition in combination with melphalan was highly effective also in MM cells lacking ongoing DNA damage. Taken together, these results suggest that MM cells rely extensively on ATR for the repair of DNA damage. We argue that ATR inhibition, alone but particularly in combination with ICL-inducing agents, may represent a novel, critically important tool to be included in the therapeutic tools used to treat MM.

ATR relieves tumor cells from replicative stress. Indeed, epithelial cancers present enhanced replicative stress and genomic instability, as a result of oncogene-driven tumultuous growth. ${ }^{29}$ We recently found that pervasive DNA damage is present in haematological cancers as well, including MM, lymphoma and leukaemia. ${ }^{19}$ To withstand this intense replicative stress and the ensuing DNA damage, cancer cells rely heavily on ATR. Along these lines, we have recently identified a subset of myeloma patients, whose cancer cells display prominent replicative stress and chromosomal instability. These patients have a dismal prognosis. MM cells presenting with ongoing replicative stress are sensitive to ATR inhibition. ${ }^{22}$ Apart from its role 

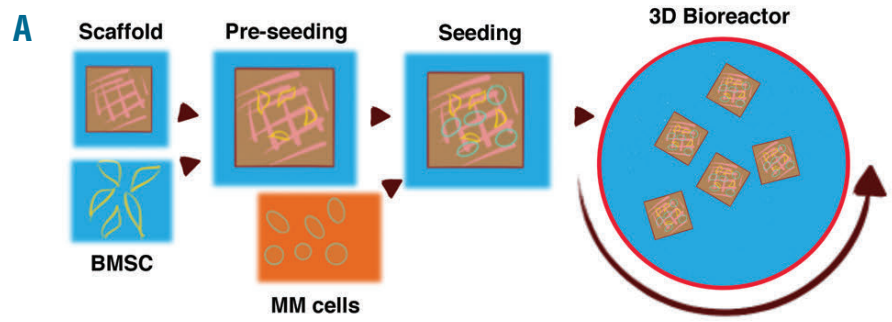

B

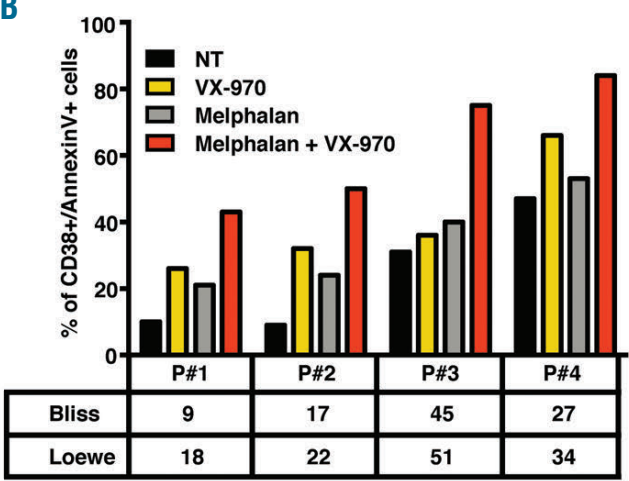

D

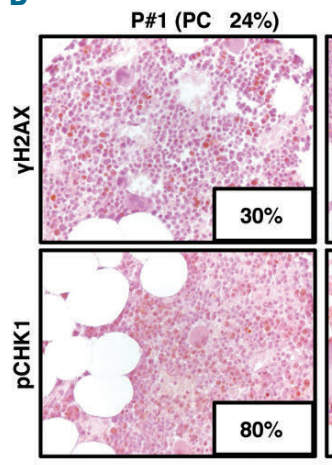

C

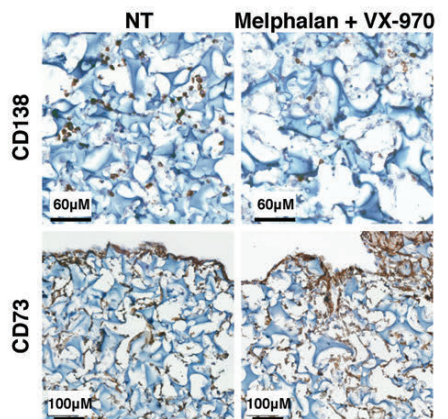

P\#2 (PC 18\%)

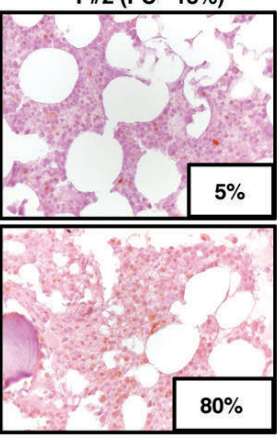

P\#4 (PC 90\%)

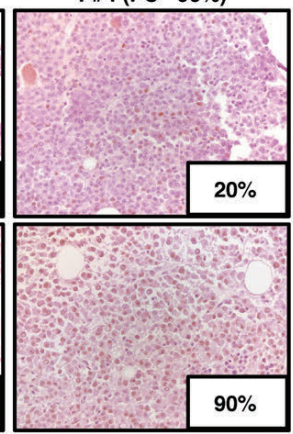

Figure 6. ATR inhibition by VX-970 synergizes with melphalan in inducing apoptosis in multiple myeloma patient cells independently of the level of DNA damage. (A) Schematic representation of the experimental procedure in the 3D bioreactor. (B-C) CD138+ primary cells from multiple myeloma (MM) patients were seeded in gelatin scaffolds pre-seeded with $\mathrm{CD}_{73^{+}}$bone marrow stromal cells (BMSC) and cultured in the 3D bioreactor system either in untreated conditions (NT) or in the presence of VX-970 $(0.3 \mu \mathrm{M})$, melphalan $(1.2 \mu \mathrm{M})$, or a combination of both drugs. (B) Seventy-two hours after the beginning of the treatment, cells retrieved from scaffolds were stained with Annexin V and anti-CD38 antibody before flow cytometric (FACS) analysis. The table underneath the graph summarizes the Bliss and Loewe synergy scores calculated for the combined treatments in each patient. Due to the limited number of cells that could be recovered from MM patient bone marrow (BM) samples, it was possible to assay just one concentration for each drug, in each patient. (C) Representative immunohistochemical (IHC) analyses performed on scaffolds populated with primary MM cells and retrieved at the end of the culture period, showing the distribution of CD138 ${ }^{+}$MM and $C D 73^{+} B M S C$ cells and the effect of the co-treatment specifically on MM cells. (D) Representative IHC images form the BM biopsies of MM patients analyzed for $\gamma \mathrm{H} 2 \mathrm{AX}$ and pCHK1 expression (original magnification 40X; samples presented are the same used for experiment in (B)). The inset in each panel indicates the percentage of tumor cells positive for the indicated marker. The percentage of neoplastic plasma cells (PC) is shown above each panel.

in replicative stress, our results suggest that the role of ATR in relieving ICL is equally important, as the inhibition of ATR strongly potentiates the activity of melphalan, a compound that induces ICL and that has been, and still is, widely used for the treatment of MM patients.

The reliance of MM cells on ATR, but not on ATM for their survival, is remarkable. ATM is pivotal for the whole cellular response to DSB. ${ }^{14}$ We and others have shown how MM cell lines as well as patient cells display exceedingly high levels of rampant DNA damage and $\mathrm{DSB}$, in the absence of exogenous DNA damage. ${ }^{19,28,30}$ Against this backdrop, and based on our data, we posit that ATR, and not ATM, is the central hub in MM cells that regulates the DNA damage response and assures the proper repair of DNA.

Based on this ATR dependency, we hence argue that $\mathrm{MM}$ cells might be particularly vulnerable to the effects of specific DNA lesions, such as ICL (where ATR is requested), while being resilient to others, such as DSB. In fact, tumors may be preferentially sensitive to compounds targeting specific branches of the DNA response. For example, drugs triggering ICL have found prominent therapeutic applications in specific cancer subtypes, which include other chronic haematological cancers such as chronic lymphocytic leukaemia and lymphomas, but also tumors of epithelial origin such as ovarian cancer, a subset of colon carcinoma and a few other selected cancers. ${ }^{31}$ In fact, when we assayed VX-970, alone or in combination with melphalan, in other two commonly used cancer tumor cell lines, cervical cancer HeLa and osteosarcoma U2OS cells, we found widely different sensitivity to these treatments (Online Supplementary Figure S14). We would then argue that a yet-to-be-characterized subset (or subgroups within) of cancer types may rely extensively on ATR for their survival, potentially being dependant on it. Additional ad hoc studies would be required to demonstrate this potential dependency.

Recently, the phosphatase CDC25A has been described as a determinant of the sensitivity to ATR inhibitors in several tumor cell lines and has been proposed as a potential biomarker to rationalize the use of ATR inhibitors in cancer therapy. ${ }^{32}$ However, it appears that CDC25A exerts a different role in $\mathrm{MM}$, as the reduced expression of the protein did not impact on the sensitivity of MM cells to the treatment with VX-970 either alone or in combination with melphalan (Online Supplementary Figure S15).

The development of resistance to ICL-inducing drugs is a pervasive plague. ${ }^{33}$ Our data confirm and expand on previous data on $\mathrm{MM}^{34}$ and other cancers, ${ }^{35}$ showing how ATR inhibition can overcome melphalan resistance in $\mathrm{MM}$. Intriguingly, a synergy between melphalan and VX-970 was evident even in cases resistant to the treatment with melphalan alone, suggesting a potential role for ATR inhibition in patients overtly resistant to ICL-inducing drugs. 
In $\mathrm{MM}$, acquired melphalan resistance has been linked to a reduction of melphalan-induced ICL, and most notably to an overall increase in the gene expression levels of the FA pathway. ${ }^{36}$ Therefore, the inhibition of ATR might supress the compensatory mechanism elicited by the increase activity of the FA pathway.

In this study we sought to identify additional synthetic tumor cells specific lethal approaches, beyond the PARP1 and BRCA1/2 axis, whereby the combination of DNA damaging agents commonly used in the clinic to treat MM could be associated with novel interventions, to prevent cells from repairing DNA and hence trigger apoptosis. We found that the inhibition of ATR was highly synergistic with ICL-inducing melphalan, strongly warranting the clinical exploitation of VX-970 in combination with melphalan as a therapy in MM patients, irrespectively of their resistance to melphalan, and the replicative stress and ongoing DNA damage of their tumor cells.

\section{Acknowledgments}

The authors would like to thank all the members of Tonon's lab for collaborative and helpful discussion and critical reading of the manuscript; Antonello Spinelli e Laura Perani from the Experimental Imaging Center at San Raffaele scientific Institute for assisting with the in vivo imaging; Raffaella Di Micco, Lucrezia Della Volpe and Anastasia Conti for sharing protocols and reagents.

\section{Funding}

Funding for this research was provided by Associazione Italiana per la Ricerca sul Cancro (AIRC; Investigator Grants and Special Program Molecular Clinical Oncology, 5 per mille no. 9965 to GT)

\section{References}

1. Puigvert JC, Sanjiv K, Helleday T. Targeting DNA repair, DNA metabolism and replication stress as anti-cancer strategies. FEBS J. 2016; 283(2):232-245.

2. Iglehart JD, Silver DP. Synthetic lethality - a new direction in cancer-drug development. N Engl J Med. 2009; 361(2):189-191.

3. Kaelin WG. The concept of synthetic lethality in the context of anticancer therapy. Nat Rev Cancer. 2005;5(9):689-698.

4. Fu D, Calvo JA, Samson LD. Balancing repair and tolerance of DNA damage caused by alkylating agents. Nat Rev Cancer. 2012;12(2):104-120.

5. Bryant HE, Schultz N, Thomas HD, et al. Specific killing of BRCA2-deficient tumours with inhibitors of poly (ADPribose) polymerase. Nature. 2005; 434(7035):913-917.

6. Farmer H, McCabe N, Lord CJ, et al. Targeting the DNA repair defect in BRCA mutant cells as a therapeutic strategy. Nature. 2005;434(7035):917-921.

7. Lord CI, Ashworth A. PARP inhibitors: synthetic lethality in the clinic. Science. 2017; 355(1):1152-1158.

8. Davies H, Glodzik D, Morganella S, et al. HRDetect is a predictor of BRCA1 and BRCA2 deficiency based on mutational signatures. Nat Med. 2017;23(4):517-525.

9. Helleday T, Petermann E, Lundin C, Hodgson B, Sharma RA. DNA repair pathways as targets for cancer therapy. Nat Rev Cancer. 2008;8(3):193-204.

10. Palumbo A, Anderson K. Multiple myeloma. N Engl J Med. 2011;364(11):1046-1060.

11. Blokhin N, Larionov L, Perevodchikova N, Chebotareva L, Merkulova N. Clinical experiences with sarcolysin in neoplastic diseases. Ann N Y Acad Sci. 1958;68(3):1128-1132.

12. Kyle RA, Rajkumar SV. Multiple myeloma. Blood. 2008;111(6):2962-2972.

13. Mateos MV, Dimopoulos MA, Cavo M, et al. Daratumumab plus bortezomib, melphalan, and prednisone for untreated myeloma. N Engl J Med. 2018;378(6):518528.

14. Blackford AN, Jackson SP. ATM, ATR, and DNA-PK: The trinity at the heart of the DNA damage response. Mol Cell. 2017; 66(6):801-817

15. Manier S, Huynh D, Shen YJ, et al.
Inhibiting the oncogenic translation program is an effective therapeutic strategy in multiple myeloma. Sci Transl Med. 2017 9(389):1-13.

16. Toledo LI, Murga M, Zur R, et al. A cell based screen identifies ATR inhibitors with synthetic lethal properties for cancer-associated mutations. Nat Struct Mol Biol. 2011;18(6):721-727

17. Belloni D, Heltai S, Ponzoni $M$, et al. Modeling multiple myeloma-bone marrow interactions and response to drugs in a $3 \mathrm{D}$ surrogate microenvironment. Haematologica. 2018;103(4):707-716.

18. Di Veroli GY, Fornari C, Wang D, et al. Combenefit: an interactive platform for the analysis and visualization of drug combinations. Bioinformatics. 2016; 32(18):2866 2868.

19. Cottini F, Hideshima T, Xu C, et al. Rescue of Hippo coactivator YAP1 triggers DNA damage-induced apoptosis in hematological cancers. Nat Med. 2014;20(6):599-606.

20. Rouzitalab M, Sani ZA, Parsaee M, Farzaneh M, Khalilipur E, Rahimi S. ATR inhibitors VE-821 and VX-970 sensitize cancer cells to topoisomerase I inhibitors by disabling DNA replication initiation and fork elongation responses. Cancer Res. 2014;74(23):6968-6979.

21. Hall AB, Newsome D, Wang $Y$, et al. Potentiation of tumor responses to DNA damaging therapy by the selective ATR inhibitor VX-970. Oncotarget. 2014; 5(14):5674-5685

22. Cottini F, Hideshima T, Suzuki R, et al. Synthetic lethal approaches exploiting DNA damage in aggressive myeloma. Cancer Discov. 2015;5(9):972-987.

23. Brown JS, O'Carrigan B, Jackson SP, Yap TA. Targeting DNA repair in cancer: beyond PARP inhibitors. Cancer Discov. 2017;7(1):20-37.

24. Hickson I, Zhao Y, Richardson CJ, et al. Identification and characterization of a novel and specific inhibitor of the ataxiatelangiectasia mutated kinase ATM identification and characterization of a novel and specific inhibitor of the ataxia-telangiectasia mutated kinase ATM. Cancer Res. 2004; 64(24):9152-9159.

25. Herrero AB, Gutierrez NC. Targeting ongoing DNA damage in multiple myeloma. effects of different Inhibitors of the DNA damage response on plasma cell survival.
Front Oncol. 2017;19(7):98

26. Rozemuller H, van der Spek E, Bogers-Boer $\mathrm{LH}$, et al. A bioluminescence imaging based in vivo model for preclinical testing of novel cellular immunotherapy strategies to improve the graft-versus-myeloma effect. Haematologica. 2008;93(7):1049-1057.

27. Xia J, Xu H, Zhang X, et al. Multiple myeloma tumor cells are selectively killed by pharmacologically-dosed ascorbic acid. EBioMedicine. 2017;1841-1849.

28. Herrero AB, San Miguel J, Gutierrez NC. Deregulation of DNA double-strand break repair in multiple myeloma: implications for genome stability. PLoS One. 2015; 10(3):e0121581.

29. Luo J, Solimini NL, Elledge SJ. Principles of cancer therapy: oncogene and non-oncogene addiction. Cell. 2009;136(5):823-837.

30. Walters D, Wu X, Tschumper R. Evidence for ongoing DNA damage in multiple myeloma cells as revealed by constitutive phosphorylation of H2AX. Leukemia. 2011;25(8):1344-1353.

31. Deans AJ, West SC. DNA interstrand crosslink repair and cancer. Nat Rev Cancer. 2011;11(7):467-480.

32. Ruiz S, Mayor-Ruiz C, Lafarga V, et al. A genome-wide CRISPR screen identifies CDC25A as a determinant of sensitivity to ATR inhibitors. Mol Cell. 2016;62(2):307313

33. Jin L, Chun J, Pan C, et al. MAST1 drives cisplatin resistance in human cancers by rewiring $\mathrm{cRaf}$-independent MEK activation. Cancer Cell. 2018;34(2):315-330.e7

34. Landau HJ, McNeely SC, Nair JS, et al. The checkpoint kinase inhibitor AZD7762 potentiates chemotherapy-induced apoptosis of p53-mutated multiple myeloma cells. Mol Cancer Ther. 2012; 11(8):17811788.

35. Kurmasheva RT, Kurmashev D, Reynolds $\mathrm{CP}$, et al. Initial testing (stage 1) of M6620 (formerly VX-970), a novel ATR inhibitor, alone and combined with cisplatin and melphalan, by the Pediatric Preclinical Testing Program. Pediatr Blood Cancer. 2018;65(2):1-11

36. Hazlehurst LA, Enkemann SA, Beam CA, et al. Genotypic and phenotypic comparisons of de novo and acquired melphalan resistance in an isogenic multiple myeloma cell line model. Cancer Res. 2003;63(6):79007906 